\title{
La Administración Pública como objeto de formación: su dimensión curricular
}

\author{
Mariana Alonso Brá \\ Magister Scientarium en Administración Pública \\ Universidad de Buenos Aires, Argentina \\ Email: mariana.alonsobra@yahoo.com.ar
}

Recepción: 30-09-2015 / Aceptación: 14-11-2015

\section{Resumen}

El trabajo puede considerarse un ensayo respecto de examinar la dimensión curricular involucrada en las propuestas de formación en Administración Pública. El componente curricular se considera desde distintos órdenes, que por la diferencia de temáticas o puntos de partida que suponen, permiten desplegarlo en distintos planos:

En primer lugar, lo curricular se examina respecto de las transformaciones que en las últimas décadas se producen en la configuración del campo de la educación superior (la centralidad que adquiere la dimensión curricular, de la mano de un progresivo protagonismo de lo educativo en el campo universitario).

Luego, el trabajo avanza sobre una definición más conceptual de lo curricular que conlleva realizar algunos análisis más detenidos dando cuenta de los distintos aspectos sociales, políticos, pedagógicos e institucionales que involucra. En este marco, se hacen presentes cuestiones significativas respecto de la relación entre producción y reproducción del conocimiento y se consideran algunas de sus implicancias de orden más operativo, en el diseño o la actualización de programas de formación.

Una vez considerado el curriculum en estos términos conceptuales más amplios, el trabajo se focaliza en dos cuestiones, propias de la especialidad de la Administración Pública, que el tratamiento más teórico de lo curricular abre:

la tensión entre lo profesional y lo académico, en tanto sesgo formativo, pero también de la propia configuración del campo de esta especialidad y, las controversias que involucra establecer cualquier tipo de ordenación entre distintas bases disciplinarias, dada una configuración multidisciplinaria, como la de la Administración Pública.

En relación con ambos aspectos, se presentan también alguna toma de posición a manera de «orientaciones deseables», de forma tal que este tipo de tensiones, entre sesgos y entre disciplinas o áreas de conocimiento, se pueda articular productivamente en una propuesta formativa fortalecida o, en correspondencia, en una conformación más amplia de la especialidad.

Palabras clave: Administración Pública, Curriculum, Formación en Administración Pública, Universidad.

\begin{abstract}
This work can be considered an assay with respect to examine the curricular dimension involved in the proposals of training in Public Administration. The curricular component is considered from different orders, other than the difference of themes or starting points that it poses, it allows to deploy them at different levels:

First, the curriculum is examined with respect to the transformations that have occurred in the last decades in shaping the field of higher education ( the centrality acquired by the curricular dimension, with the help of a progressive role in the educational university field ).

Then the work proceeds on a more conceptual definition of the curriculum involved in making some more detained analysis realizing the various social, political, educational and institutional aspects involved. In this context, significant issues are present on the relationship between production and reproduction of knowledge and consider some of its implications more operational order, in designing or updating training programs.
\end{abstract}

Once considered the curriculum in these broader conceptual terms, the work focuses on two issues of the specialty of public administration, that the theoretical treatment of the curriculum opens :

- The tension between the professional and academic training as bias, but also the configuration of the field of this specialty and,

-Disputes involving establish any disciplinary ordination between different bases, given a multidisciplinary settings such as Public Administration.

For both aspects, any position taken by way of "desirable orientations" are also presented so that this kind of tension between bias and between disciplines or areas of knowledge can be articulated productively in a training proposal or strengthened correspondingly wider in specialty conformation.

Key Words: Public Administration, Curiculum, Taining in Public Administration, University. 


\section{Introducción}

El propósito fue examinar la dimensión curricular involucrada en la definición de propuestas de formación sobre la Administración Pública. Esta dimensión es considerada desde diferentes puntos de partida que intentan recoger y analizar los aspectos que consideramos abiertos (incluso polémicos) respecto de la definición de programas de formación en esta especialidad.

Estos aspectos organizan el trabajo en cuatro temas-cuestiones, aparentemente aisladas pero derivados de la centralidad dada al curriculum:

a. El examen de lo curricular, en un sentido general, respecto de las transformaciones que en las últimas décadas se producen en la configuración del campo de la educación superior.

b. El tratamiento del curriculum en tanto aporte teórico del campo educativo, que permite realizar algunos análisis más detenidos para delimitar en nuevos términos los distintos aspectos sociales, políticos, pedagógicos e institucionales que involucra.

c. La tensión entre lo profesional y lo académico, constitutiva de la Administración Pública, en tanto sesgo formativo, pero también de la propia configuración del campo de esta especialidad y,

d. las controversias que involucra establecer cualquier tipo de ordenación entre distin- tas bases disciplinarias, dada una conformación multidisciplinaria.

El valor de estos aspectos en relación con el tratamiento de las propuestas formativas, no es conclusivo, ni prescriptivo, ni descriptivo; es decir, su aporte primordial consiste en vincular dimensiones aparentemente aisladas o ajenas al tratamiento de lo curricular, abriendo el escenario sobre el cual pensar respecto de este objeto de estudio y de formación.

\section{Educación Superior y curriculum}

En la última década, en el campo de la educación superior, resultó creciente la preocupación por la currícula, entendida como un plan de «contenidos» sistematizados y organizados para considerar la enseñanza, en tanto propuesta formativa.

Esta preocupación de las instituciones de educación superior, en principio, apuntaba a la problemática de la «actualización» de la formación que se brindaba en este nivel, en relación con cambios rápidos y pronunciados en muchos aspectos de la vida social y económica que, según se consideraba, merecían atención a través de una revisión profunda del tipo de formación que se ofrecía, en distintos campos disciplinarios $y$, particularmente, profesionales.

Aunque, esto último conllevó comenzar a instalar la renovación curricular en términos de una intervención permanente. Es decir, el interés por la actualización, especialmente concentrado en las propuestas tradicio- 
nales de grado, derivó también en una nueva percepción de que estos planes y programas, independientemente de los proyectos de reformas curriculares que se iban generando progresivamente en términos de "actualización», debían tener una atención regular ad hoc.

Así, en el caso de la educación universitaria argentina, sobre el final de los años 90, $y$ en el marco de fuertes transformaciones de política universitaria (con concreciones tanto en nuevas definiciones ministeriales como en proyectos de las propias instituciones), el panorama era bastante convergente respecto de iniciativas de reformas curriculares en marcha: creación de áreas institucionales específicas, aparición de programas de posgrados dedicados a la cuestión de la enseñanza superior, generalización de diseños innovadores flexibles, determinación de parámetros comunes inter -institucionales, identificación de indicadores de «calidad» disciplinaria o profesional, etcétera. (Fernández Lamarra, 2003).

Iniciativas diversas que se presentaban desde distintos niveles y con diferentes alcances: Consejos Universitarios, Rectorados, Asociaciones de Facultades, Secretarías, Departamentos Académicos o, incluso, Programas, Carreras o Cátedras y que de una u otra forma cubrían el conjunto universitario nacional. Un panorama, en términos generales, que podría identificarse también presente; si bien con sesgos nacionales específicos, en la actividad universitaria de América Latina, durante esta década pasada y con presencia cierta en la actual.

Sin duda, esta emergencia de la cuestión curricular se trama con otras cuestiones de mayor alcance que involucran la propia reconfiguración de la formación universitaria, del amplio espectro institucional involucrado con ella, de sus dinámicas de crecimiento y de sus relaciones. (Fanelli, 1996, 1998)

Entre otras, cabría considerar su re- definición en términos más amplios de Educación Superior, agregando diferentes tradiciones formativas, y generándose paulatinamente una formación de nueva índole, donde la educación de último nivel, ya no es espontáneamente ligada a una significación bastante compartida y estable respecto de la naturaleza y las condiciones de lo universitario. ${ }^{1}$

Una política que tiende a establecer equivalencias entre la educación no universitaria (terciaria) y universitaria, generándose paulatinamente una indiferenciación progresiva como educación de último nivel, indistinta. Incluso, las propias universidades fueron incorporando propuestas hasta entonces propias de aquel tipo institucional no universitario: carreras cortas, de "pre-grado", de fuerte contenidos técnicos para una pronta inserción laboral. Es decir, la identidad institucional de las universidades se redefine en estos otros términos más amplios de «Educación Superior».

1. En sentido estricto, la consideración primera de un ámbito de Educación Superior, es previo y puede ubicarse en los años 60, en el marco de un pasaje de la Universidad de elite a la Universidad de masas; por lo menos en el caso de los países centrales, especialmente los europeos (Krotsch, 2000b). En la década de los 90 adquiere una consolidación definitiva en tanto tal, especialmente al ser considerado como contenido explícito de políticas públicas gubernamentales y de organismos internacionales. 
Consonante con este proceso de redefinición de lo universitario, es el proceso de masificación. Si bien se trata de uno lento, en proceso ya desde inicios de los años 70 (mientras que en Europa en la década previa), y sólo interrumpido por períodos, especialmente en las dictaduras de los países del Cono Sur. ${ }^{2}$ Sobre el año 2000, tiene presencia como tendencia sólo en términos de cantidades absolutas y no de progresión en términos relativos. El crecimiento se presenta en cambio negativo, aún en términos absolutos para algunos campos (Klein y Sampaio, 1996; Fernández Lamarra, 2003). ${ }^{3}$

Pero avanzada la década de los 90, el aumento de la matrícula da lugar a numerosos estudios acerca del fenómeno de «masificación» en tanto fundamento de muchas de las transformaciones de política universitaria. Entre ellas, el crecimiento de la cantidad de instituciones, especialmente de aquellas de carácter privado. Tendencia muy significativa respecto de la transformación del conjunto.

Esta desregulación de facto $^{4}$ respecto de la creación de nuevas universidades, generalmente fue sucedida de una regulación de nueva naturaleza que alcanzó al conjunto (equiparándolo), tanto a las tradicionales estatales como a las recientes privadas, sobre el principio de «libre concurrencia». Un nuevo sentido de la regulación estatal que se construye también con criterios diferentes del tradicional prestigio académico-disciplinar.
Las políticas de evaluación y acreditación son paradigmáticas en este sentido e incorporan criterios propios de una expertez educativa donde la dimensión curricular es uno de los aspectos centrales de definición y evaluación de una propuesta formativa universitaria (Dias Sobrinho, 2002).

Aquel rápido crecimiento de instituciones desde el ámbito privado y sobre matrices y condiciones de lo más variadas, sucedidas de su reconocimiento formal estatal, en relación con las nuevas modalidades de regulación resultan en transformaciones muy grandes: de aquí en más es posible «ser» universidad $\mathrm{u}$ ofrecer formación superior de muy diversas y variables formas, con una legitimidad en el campo académico que comienza a ser relativamente equivalente para las más variadas propuestas (Alonso Brá, 2008).

Desde este escenario, la flexibilización curricular - considerada desde múltiples aspectos - alcanza su mayor despliegue: qué tipo de organización académica se corresponde a determinado diseño curricular, qué se consideran «contenidos» de enseñanza; qué lugar y qué relación le cabe a la investigación en programas formativos; qué rango de divergencias entre perfiles de graduados en campos equivalentes es aceptable; cómo se unifican/segmentan trayectos; qué se consideran circuitos iniciales o terminales, que tipo de evaluación y seguimiento son aptos; cuál es el nivel de estructuración/apertura de los planes, entre otros.

2. En estos períodos, en casi todos los casos nacionales, se da un decrecimiento en términos relativos y absolutos.

3. Proyecciones para esta década respecto del caso argentino, por ejemplo, anuncian en cambio su decrecimiento relativo. 4. Por ejemplo en el caso argentino, mucha de las instituciones universitarias que son creadas en la primera mitad de los años 90 , se encuadran en una condición de «excepción», a la legalidad establecida, vigente aún en ese momento, respecto de décadas previas. 
De esta forma, comienza a ser posible desarrollar un programa de formación con una modalidad particular - específica -, diferenciándose dentro de una misma institución o comunidad disciplinaria, antes que atendiéndose a un modelo de referencia para emular, dado tácitamente en el tiempo, por ese encuadre institucional, disciplinar o de la especialidad. La dispersión es más fuerte en el nivel de postgrado, dada su expansión y generalización más reciente en la Región, salvo en los casos más restringidos donde tiene una institucionalización más temprana (v.g. el campo académico brasileño, en los años 70).

Finalmente, una tendencia importante es la emergencia de una preocupación creciente por la enseñanza en términos de propuestas educativas claramente delimitadas en su especificidad y con inserción cierta y validada en el mundo ocupacional o productivo; es decir, de un marcado carácter profesional y aplicado, antes que académico en sentido estricto.

Un indicador de esta tendencia más general puede verse en otra derivada, que resulta en re-colocar la enseñanza en sí misma, en un lugar neurálgico del campo universitario, respecto de la tradicional centralidad excluyente de las disciplinas y respecto de la consiguiente importancia de las actividades de investigación, en tanto fundamento indispensable del soporte y crecimiento de aquellas. Investigación que entonces se consideraba como creación propia de este campo académico (de carácter intelectual- cultural o científico, según la especialidad disciplinaria de referencia) y, por lo tanto, no condicionada respecto de otros requerimientos diferentes de aquel (por ejemplo, los provenientes de otros campos como el productivo).

La disciplina y lo académico y, por extensión, la investigación -en tanto elemento constitutivo de aquellos- que históricamente configuraban lo universitario, en tanto producción propia, ad hoc, excluyente, que hacían a su autoridad social, a su condición de institución social, van erosionándose progresivamente (Gibbons, 1999; Krotsch 2000a); adquiriendo centralidad el carácter «educativo» de la actividad universitaria. Y en un mismo sentido el desplazamiento de la investigación hacia la investigación «aplicada»e, incluso, el desarrollo tecnológico. En cualquier caso, se trata de lo universitario puesto en cuestión y, entonces, de un desplazamientos hacia otros campos diferentes que actúen como fundamento, como soporte de su pertinencia social (la educación, la tecnología, etc.).

A partir de aquí, entonces, lo curricular se presenta como un área de especialidad «educativa», capaz de desplegarse sobre distintos ámbitos disciplinarios e institucionales. Esto es posible porque lo educativo adquiere una legalidad propia en el campo universitario, independiente y en tensión respecto de la legalidad disciplinar antes claramente predominante.

Esta tendencia se vincula con la centralidad de la formación profesional y las numerosas iniciativas de integración económica con lo que ellas conllevan en términos de establecer denominadores comunes, para la circulación y movilidad entre espacios nacionales. En su 
base están los procesos de globalización, ya sea considerados en términos económicos como sociales, sobre los que transcurre una realidad formativa crecientemente tras- nacionalizada, pero también fuertemente diversificada y segmentada, con dificultades de regulación. El caso de la Comunidad Europea resulta bastante ilustrativo en este último sentido, especialmente a través del desarrollo de un conjunto de iniciativas muy operativas y en plena marcha, susceptibles de ser consideradas dentro de lo educativo-curricular: el establecimiento de procedimientos comunes para la «traducción» a competencias de las diversas titulaciones finales y la producción de otros denominadores comunes como los créditos educativos, para unificar los más variados trayectos de formación, en el marco de la movilidad internacional de graduados y de estudiantes, respectivamente.

En cualquier caso, es posible identificar como lo universitario sufre un cambio radical y ya para fines de los años 90 queda claramente re-interpretado en términos educativos $y$, en relación curriculares. $\mathrm{O}$, en otras palabras, como la cuestión curricular gana protagonismo y está vinculada a cambios estructurales, políticos-sociales y económicos globales.

\section{Desarrollo}

\section{El curriculum qua relación entre un texto y un contexto}

En el apartado previo, se presentó un panorama general de la educación superior y de sus implicancias en términos curriculares.
También se consideró la vigencia de cuestiones de tipo de educativo, como los procesos formativos mismos y como esto involucraba un cambio importante que se sobre-imprimía a una tradición universitaria más bien asentada sobre la cultura académica y disciplinar.

A ciencia cierta, los desarrollos conceptuales en materia curricular parten y mantienen una vinculación casi excluyente con el campo educativo y, por ello, a los procesos de escolarización, mirados desde su faz tanto política y social como didáctica. El estudio de lo curricular situado (conceptualmente) en el campo universitario es poco frecuente y no cuenta aún con una producción significativa que altere alguna de las cuestiones claves donde se asienta el estudio de lo curricular (hasta ahora situado, expresa o tácitamente, en el espacio escolar).

En general, tienen como referencia primaria un proceso de «enseñanza», el escolar, y unos sujetos (maestros y alumnos) que no son linealmente asimilables a sus pares universitarios ya que, en última instancia, escuela y universidad son instituciones sociales diferentes, con condiciones históricas de emergencia, tradiciones, valores mitos y reglas de juego todavía cualitativamente distintos.

Sin embargo, también es posible establecer ciertas convergencias significativas o traslados válidos, en tanto lo universitario, tampoco pudo pensarse totalmente por fuera de lo formativo. Maestro-discípulos es una figura que siempre estuvo de alguna manera recreada en este ámbito y que aún mantiene vigencia cierta en la vida universitaria. 
Por otro lado, es bastante generalizada su condición de institución que provee socialmente determinados saberes y constituye determinados sujetos y roles sociales. Lo que, más allá de las características específicas que adopta en el espacio universitario (conocimientos más especializados, más complejos o con una distribución más restringida), la hace equiparable a la educación formalizada en su conjunto.

A su vez, en el caso de nuestras universidades latinoamericanas, tempranamente orientadas a la formación en profesiones liberales, antes que al desarrollo científico propio de instituciones de corte humboltiano, este sesgo formativo se pronunció y se continúa pronunciando.

Nuestro propósito ahora es partir de algunas consideraciones teóricas, propias del campo educativo, para situarlas en el campo universitario o en el de la educación superior en general, de forma de ampliar la definición previa dada a lo curricular y de reflexionar sobre sus implicancias en el diseño y desarrollo de Programas abocados a la Administración Pública.

En general, todos los aportes teóricos en el estudio del curriculum consideran como requerimiento básico en su constitución, la existencia de la delimitación y organización de un conjunto, definido como reunión de conocimientos, de saberes, de contenidos, de prácticas o experiencias, según el tipo de perspectiva; pero siempre se trata de un conjunto institucionalizado.

Es decir, el curriculum en tanto tercer elemento que se diferencia de la relación docen- te- estudiante debe de estar de alguna forma «establecido», definido, recortado en términos de una prescripción de algún tipo (aunque no necesariamente explícita), que no deja librada la enseñanza o el aprendizaje a la espontaneidad, la libertad, o el transcurrir incierto de esa relación.

El concepto de curriculum siempre supone un componente sustantivo de estructuración o formalización, esté dado (menos visible pero más profundamente) por la trama cultural y política organizacional en la que se desarrolla la relación educativa o, más linealmente, a partir de documentos normativos; según los énfasis relativos que se consideren. Es decir, para que haya curriculum debe haber alguna institución mediando y constituyendo la relación a partir de este tercer elemento.

Para ponerlo en otros términos, nunca podríamos hablar de curriculum en aquella universidad medieval de Bologna donde se reúnen casi ociosa y espontáneamente maestros y discípulos; ni en relación con el Emilio rousseauniano que aprende libremente en «estado de naturaleza».

Así, la modernidad con la escolarización universal es un punto de referencia obligado (histórico o abstracto) desde el cual parte cualquier desarrollo conceptual para la comprensión de lo curricular:

Se instala un nuevo sistema escolar que se diferencia del anterior en el reclutamiento del alumnado, los fines de la educación y la selección de contenido (...) Los conoci- 
mientos y destrezas objeto de aprendizaje dejan de estar determinados por el contexto inmediato; más bien hay que abstraerlos de dicho contexto, e introducirnos en un texto (...) dicha selección tiene que ser organizada y deberán organizarse formas de enseñanza. (Lundgren, 1992: 30-31).

Lo curricular puede considerarse entonces en términos de un proceso social y político que conlleva esta abstracción e introducción, cierto violentar un ámbito (el contexto social, de socialización más «espontánea»o «natural») para constituir otro de nuevo carácter («un texto», la institución escolar, el curriculum). Pero se trata de un texto o conjunto estructurado que permanece en alguna medida sujeto a aquel primero, en tanto su génesis se explica como pasaje, traslación o transposición. Es decir, para definirse y fundamentarse no puede abandonar aquel del cual parte y se diferencia.

El curriculum entonces puede interpretarse en un sentido más global y abstracto como una relación compleja, instituida de alguna forma socialmente, entre un texto (el curriculum, en sentido estricto) y un contexto (el primer ámbito a partir del cual se configura y que le provee sentido):

La búsqueda de formas de articulación entre los contextos sociales de producción y reproducción se constituye en el problema fundante del curriculum y éste designa entonces el conjunto de textos elaborados para solucionar el problema de la representación de los saberes y des- trezas generado por la separación de ambos contextos (Col; 2001:54).

Así el «contexto» social o, con más precisión, lo «exterior» al curriculum se presenta como el ámbito propio de producción y generación de saberes, conocimientos, destrezas, cultura, etc. Mientras que, la enseñanza organizada en él (el interior, el texto) como un ámbito estructurado respecto de la reproducción social del primero.

En este punto sería necesario hacer una primera diferenciación respecto de la educación formalizada y de la universidad. La primera, históricamente se conformó como un lugar pleno de reproducción: una instrucción sobre valores y conocimientos ya dados, necesariamente pre-establecidos, que sólo se debían transmitir, inculcar o generalizar en forma universal, de acuerdo a determinados procedimientos normados.

Lo señalado explica muchas de las limitaciones y contradicciones que afronta la escuela en la actualidad, cuando se espera de ella otro tipo de función social (v.g. la construcción de una relación no «alineada» o autónoma de los docentes con el conocimiento, objeto de enseñanza, como requerimiento de un aprendizaje significativo). Este tipo de preocupaciones pone en jaque su sentido más histórico $y$, en primer término, este problema de representación o de transposición entre texto y contexto, o entre contextos diferenciales, fundacionalmente sesgados en profundidad. 
Esta cuestión, en alguna medida, esta presente también en los institutos de educación terciaria, no universitaria, por su filiación en general más directa a los sistemas educativos y sus características constitutivas.

Pero lo universitario, en cambio, esta configurado como una institución socialmente reconocida como productora de conocimiento, es decir, conviviendo ella ambos contextos, con predominios variables. En este caso, la relación entre texto y contexto adquiere aristas particulares y, en alguna medida, parcialmente se traslada al propio espacio institucional en términos del tipo de relación construida entre la enseñanza y la investigación. Relación no sólo considerada en términos de ámbitos diferenciados funcional u organizacionalmente, sino en su articulación más general respecto del sentido de la actividad universitaria y del perfil formativo y disciplinar-académico de los sujetos que la llevan adelante.

En decir, en términos universitarios, la reflexión sobre lo curricular involucra un examen detenido de esta relación entre contextos de producción y de reproducción, respecto del propio ámbito institucional, sea éste considerado como el establecimiento donde se inserta o como las comunidades disciplinar o interdisciplinares sobre las que se despliega la especialidad y sus organizaciones específicas. Distintos planos en los que puede pensarse el propio ámbito institucional, con una vinculación variable al espacio universitario.

Traducido a términos más operativos, la definición curricular de un programa de formación requiere dilucidar en qué medida, de qué forma y en qué grado se generará esta escisión o transposición texto - contexto, propia de cualquier propuesta formativa institucionalizada.

Ello involucra definir algunas cuestiones sustantivas, que en general se presentan en términos de tensiones y, por lo tanto, conllevan tomar posiciones relativas. Entre las referidas a los contextos de producción cabría señalar:

- el carácter disciplinar o interdisciplinar del objeto de formación y su composición relativa (en tanto contexto de producción, delimitado sobre parámetros tradicionalmente académicos).

- el carácter transdisciplinar del objeto de formación (en qué medida su contexto de producción predominante no se puede adscribir ya a un conjunto determinado de disciplinas y se presenta con una configuración totalmente diferenciada y específica).

- la ecuación particular entre su carácter profesional/ académico (en qué medida el contexto de producción se extiende por fuera del ámbito académico, disciplinar o universitario e involucra otras organizaciones sociales tales como corporaciones profesionales).

En el campo de la Administración Pública, ninguna de las dimensiones y categorías consideradas tiene un sentido excluyente, con lo cual, estas definiciones resultan particularmente complejas y requieren de acuerdos o 
convenciones relativamente compartidas. Sobre esta complejidad, a su vez, se extienden no sólo diferentes sesgos institucionales, académicos, disciplinarios y profesionales, sino también (y en relación) diferentes concepciones y posiciones teóricas e ideológicas, en tanto especialidad que podría considerarse legítimamente dentro de las ciencias sociales, las que se conforman y desarrollan, justamente, en esa pluralidad controvertida.

Es decir, el proceso de abstracción, introducción y selección en tanto construcción de un «texto» o de un curriculum en sentido estricto, requiere previamente la determinación tentativa, precaria y seguramente arbitraria, de un «contexto de producción» que, en el caso de la Administración Pública, de por sí, se presenta en movimiento, diverso y variable, es decir, que no se encuentra claramente delimitado, estabilizado o plenamente institucionalizado. ${ }^{5}$

Por su parte, la construcción del texto, en sí mismo, está directamente ligada a la reconstrucción del contexto de producción que se haga para tal fin. Es decir, la arbitrariedad inherente a esta re-creación (la determinación de los distintos ámbitos académicos, disciplinarios, profesionales o sociales desde donde se produce conocimiento relativo al objeto Administración Pública) se traslada a la producción misma del texto: en principio, cuáles son los «contenidos» válidos o pertinentes para la formación en esta especialidad, en tanto primera operación de abstracción y selección.
Lo que queremos destacar es que, frente a la situación de definir un programa de formación, la propia reconstrucción inicial de los contextos de producción es arbitraria, ya involucra en sí misma este procedimiento de abstracción y selección. Es materialmente imposible determinarlos o aprehenderlos en un sentido absoluto, en su totalidad, independientemente de sesgos, concepciones, trayectorias institucionales o posiciones; esta determinación siempre conlleva un recorte y una definición de algún tipo (o varias contradictorias incluso), respecto de las cuales el encuadre institucional, a partir del cual se desarrolla el programa, es muy significativo.

Para ponerlo en otros términos, la separación entre contexto y texto es más bien abstracta, no tiene una expresión operativa, instrumental, directa en la realidad. Y esto ocurre porque el establecimiento de ese contexto (dónde se concreta la producción social de conocimientos, saberes, experiencias en relación con determinada especialidad) es una demarcación siempre dada desde la posición o la inquietud por definir el texto (el programa de formación) y no a la inversa.

Sin embargo, la legitimidad del texto debe estar anclada en un contexto. Como señalamos previamente, se trata de un texto "sujetado", que para fundamentarse o legitimarse en su validez o pertinencia no puede abandonar aquel contexto del que se diferencia (el conocimiento social mismo o dónde este se pro-

5. Las tendencias a las que aludimos en las secciones previas, respecto de las transformaciones en la configuración de lo universitario, sin duda, también están presentes respecto de este escenario de dispersión. 
duce) porque su propósito es sólo su reproducción a través de la enseñanza.

Aunque, complicando un poco más las cosas, sí podríamos afirmar que ese texto, una vez institucionalizado, comienza a formar parte de ese contexto. Es decir, un programa de formación siempre tiene una incidencia (aunque variable) en la producción social de conocimiento, ya sea porque se lo proponga directamente o porque ya supone e instituye una configuración específica del campo de la especialidad a través de su enseñanza (determinadas concepciones, sesgos, perspectivas, conocimientos, etc.).

Nos equivocamos si pensamos demasiado en el curriculum como una cosa o un objeto y no en tanto idea, símbolo, institución cultural. Las instituciones son el necesario contexto para la deliberación y la acción, son los vehículos a través de los cuales el curriculum se vuelve real; pero además el curriculum es en sí mismo una institución cultural y social (Reid, 1999: 12).

En este último sentido, el texto, el curriculum o con más precisión, esa relación interiorexterior que supone, también es productora de conocimiento, justamente por el carácter arbitrario de construcción que conlleva: determinado recorte estructurado e institucionalizado de un campo mucho más vasto y; de alguna forma, indeterminado (el contexto social o cultural) al que simultáneamente aporta y co-constituye.
Así, por ejemplo, un desarrollo y una expansión rápida y generalizada de programas de formación en Administración Pública involucrarían un crecimiento y fortalecimiento de esta especialidad, en relación con otras; y probablemente la emergencia de nuevos ámbitos de producción vinculados, o una influencia mayor del tipo de preocupaciones propias de la especialidad en otras disciplinas o áreas de conocimiento vinculadas (v.g. la sociología, la administración, el análisis organizacional, etc.). A su vez, un sesgo por caso más politológico que administrativista en el diseño de estos programas en crecimiento, como dirección predominante, seguramente involucraría una tendencia relativa a reconfigurar el conocimiento de la especialidad en estos nuevos términos (respecto de otros posibles). Y, así podríamos continuar con nuestro ejemplo, respecto del carácter más académico o más profesional, respecto de perspectivas teóricas o ideológicas, etcétera.

Pero, retomando nuestra preocupación más operativa, el diseño del texto, del programa, también involucra definir algunas cuestiones en términos de una toma de posición, vinculada con la relación producción - reproducción de conocimiento en el ámbito universitario y del propio programa:

- Cuál es el perfil docente predominante: en relación con su extracción disciplinaria o interdisciplinaria respecto de la especialidad, en relación con su condición académica- profesional y directamente vinculado, en relación con su condición 
de productor de conocimiento o del diálogo autónomo que produce con él (no fijado, en la sola reproducción), a partir de la práctica de enseñanza.

- Cuál es el perfil de estudiante y graduado deseable: en relación con la definición de expectativas profesionales- académicas en juego, tanto respecto de su incorporación, su proceso formativo y su egreso e inserción posterior. También, respecto de la relación que entable con el conocimiento, según el tipo de vinculación con la propuesta docente, más reproductiva o más autónoma.

- Cuáles son los contenidos y cómo organizan y estructuran la relación educativa.

En principio qué es susceptible de ser considerado como contenido válido: la transmisión de experiencia acumulada y de información actualizada, dada una trayectoria profesional reconocida (asociadas a las figuras docentes) o la producción más sistematizada de distintos campos disciplinarios o interdisciplinarios de carácter teórico o conceptual (asociadas a figuras o grupos típicamente académicos o intelectuales). Cuáles se consideran dimensiones de estudio de la especialidad, cuáles se privilegian, cuáles excluyen. Cuáles son los criterios que delimitan estas dimensiones, de qué tipo (en qué medida disciplinar-interdisciplinar, en qué medida de desempeño profesional o en qué medida dados por ámbitos institucionales vinculados a la especialidad).
Qué grado de convergencia o dispersión, de unidad o de flexibilización pueden adoptar la estructuración general del curriculum en tanto trayecto formativo, y en relación con esas definiciones más generales respecto de los contenidos y de los perfiles de docentes y de estudiantes. A su vez, qué margen de autonomía queda librado a las definiciones de docentes y estudiantes respecto de esta formalización prescripta. Cómo se establecen secuencias, ciclos $\mathrm{u}$ orientaciones dentro del Programa (cómo se corresponden con la situación de desarrollo de la especialidad y los sesgos específicos definidos) Qué organización académico- institucional en términos de áreas y responsables requiere la estructuración definida. Cuál es el alcance de su intervención respecto de docentes y estudiantes o de su relación formativa.

Estas definiciones deberían ser consideradas tanto en términos de diseño, primero como de curriculum en movimiento; es decir, de procesos que se van desarrollando en el tiempo y que pueden conllevar re-definiciones sucesivas (aunque no necesariamente progresivas). En otras palabras, no sólo en un sentido racional-planificado, sino por la propia dinámica de relaciones y prácticas de actores individuales e institucionales, directa o más indirectamente involucrados en él.

Como indicamos previamente, las definiciones curriculares pueden considerarse sobredeterminadas por las adscripciones institucionales donde se encuadra el Programa. Una cuestión que en los desarrollos teóricos sobre 
curriculum se denomina "campo recontextualizador», de construcción del «texto privilegiante» (Bernstein, 1993).

Este campo es el que estaría jugando en relación con la arbitrariedad que supone la definición del contexto primero, social o productor y en relación con la arbitrariedad del recorte sobre aquel, que conlleva el curriculum (el proceso de abstracción, de introducción y de selección de conocimientos, transpuestos a contenidos de enseñanza). $\mathrm{O}$, en términos más propios de este autor, sería el conjunto de organizaciones, agentes, grupos, especialistas que operan a través de determinadas tecnologías, dispositivos o procesos para regular la circulación de textos (o discursos) entre el contexto primario (social, productor) y el secundario (curricular en sentido estricto, de reproducción).

En este campo re-contextualizador (que media entre ambos contextos o entre un texto prioritario y su contexto) podemos incluir el conjunto de agentes y dispositivos tratados en relación con la reconfiguración del campo universitario en la historia reciente: la política pública del sector y las modalidades de gestión específicas que asume; la presencia de mecanismos de regulación y posiciones de actores propios de la constitución de relaciones competitivas o de tipo mercantil; la creciente presencia de actores organizacionales del ámbito productivo o profesional como referencias significativas para orientar la actividad universitaria; la progresiva incidencia del campo educativo (sus especialistas y sus tecnologías) para comprender e intervenir en la actividad universitaria, etcétera.

Aunque también podemos considerar esta recontextualización dada por la adscripción institucional directa que tenga el Programa - es decir, el tipo de actores individuales o colectivos de ese marco - sus relaciones, sus proyectos e inserciones disciplinarias, académicas, político-académicas, profesionales, etc.

En general, una red de relaciones comprensible en términos de poder y de cultura que estructura y configura la institución más amplia donde se inserta el Programa (v.g. universidad, facultad, Departamento, etc.). Pero, especialmente, el Programa, una vez conformado, como unidad organizacional, puede comprenderse en éstos términos equivalentes de una estructuración institucional específica, particular (articulada con la previa) que opera también con un sentido re-contextualizador más próximo.

Finalmente cabría señalar que en ningún caso, las definiciones respecto del «texto privilegiante» que produce este campo mediador son unívocas o taxativas. Involucran siempre posiciones relativas, que creemos pueden considerarse primariamente organizadas respecto de la tensión producción-reproducción, como constitutiva de lo curricular.

Esta tensión puede ser considerada en términos más concretos respecto del tipo de relación que establezca el programa entre actividades de enseñanza-investigación (v.g. en qué medida esta última está contenida en la rela- 
ción educativa, a través de la definición del perfil de docente, del perfil de estudiante o de la selección y organización de los contenidos o trayectos).

Pero, además, puede ser apreciada en un sentido más abstracto, respecto de tipo de propuesta formativa: sea que ésta involucre una concepción de la enseñanza o del aprendizaje, como una transmisión/adquisición de un saber dado, clausurado externamente (propio y exclusivo de ese contexto ajeno que sólo se reproduce), o de diálogo reflexivo o crítico con ese saber, de base más tradicionalmente académica (donde producción y reproducción pueden intersectarse, por lo menos en cierta medida, en un mismo espacio).

En las secciones siguientes se analizará con mayor profundidad dos cuestiones presentes en la definición del «texto privilegiante», considerando el caso particular de la formación en Administración Pública: el carácter profesional/académico de su «objeto» y su condición disciplinar/transdiciplinar.

Se trata de dos aspectos que, si bien no cubren desarrollándolo el conjunto de preocupaciones planteadas respecto de la producción del curriculum, resultan particularmente significativos para reflexionar sobre la formación en esta especialidad, dadas sus características específicas.

\section{La Administración Pública: entre la disci- plina, la multi-disciplina y la profesión}

Detenerse en la relación entre la Administración Pública y un programa de formación universitaria en la materia, sitúa en la reflexión sobre el carácter de la relación entre la práctica de la gestión pública y el desarrollo propiamente académico, formativo.

Esta vinculación consiste en abordar la gestión pública, en tanto práctica social (predominantemente de condición estatal en nuestra Región) para transformarla en objeto desde el campo académico; es decir, en materia de estudio, problematización y análisis; cuando aquella - en cambio - se presenta con las exigencias propias de una actuación e intervención directa para la resolución de problemas, de orden pragmático $\mathrm{y}$, muchas veces, incluso de urgencia política o social. En otras palabras, esta vinculación conlleva transfigurarla desde un plano pleno de «sujeto y práctica activos» a un «objeto pasivo», con un nuevo sujeto que opera sobre él y, en alguna medida, la reconforma: el académico, el docente, el investigador.

La vinculación planteada también puede leerse en los términos ya tratados de una relación entre contextos diferenciales, en tensión, propia de la dimensión curricular de las propuestas educativas: un ámbito social, de prácticas y actores específicos, que debe trasponerse en objeto de estudio y de formación, dadas determinadas regulaciones. 
La escisión entre ambos contextos (el propio de la administración pública, en tanto práctica profesional; y el académico, en tanto, espacio reflexivo respecto de ella) se expresa en la distancia de los requerimientos y prioridades de uno y otro:

Transformar la gestión pública en materia de examen intelectual o académico conlleva introducir la incertidumbre, desnaturalizar supuestos, construir cuestiones, abstraer procesos en términos conceptuales, debatir y confrontar con otras producciones de la especialidad, desde distintas tradiciones teóricas o ideológicas. En definitiva, asumir una perspectiva crítica en términos de abrir preguntas, tomando distancia de la gestión misma para reconstruirla como objeto de análisis.

La práctica de la gestión, en cambio, requiere la inserción directa en el campo, la certeza o la reducción de la incertidumbre, la identificación y el seguimiento de las «buenas prácticas» en la materia, la información precisa; la producción de respuestas seguras y simples frente a las distintas cuestiones que se presentan, que permitan, a manera de reaseguros, involucrarse plenamente para la «decisión» o la «acción».

En síntesis, una cuestión significativa que debe resolver el diseño de un programa de formación en esta especialidad es: en qué medida va a estar dirigido a constituir un «buen funcionario» con competencias directamente ligadas a la administración pública como práctica profesional-ocupacional y en qué medida a construir este campo de actividad social, como objeto de estudio y de desarrollo teórico o reflexivo, no inmediatamente instrumental a las lógicas predominantes en ese campo, en tanto espacio de actuación.

Esta tensión puede ser traducida a los términos de reflexión versus prescripción o, también a los de academia versus gestión; y constituye un asunto importante y bastante significativo para la Administración Pública que se construye como una especialidad híbrida, respecto de su condición compartida de especialidad académica y actividad profesional.

La hipótesis es que esta hibridez - a diferencia de otro tipo de especialidades que se constituyen simultáneamente en ambos ámbitos (profesional y académico)- en el caso de la Administración Pública, se presenta en términos de una tensión intensa que dificulta su articulación cierta: una formación asentada en la reflexión de carácter plural - propiamente académica - para consolidarla en objeto de conocimiento cultural y socialmente pertinente que, a su vez, permita una comprensión más profunda del campo de actuación para intervenir sobre él de manera más crítica, más aguda y fundada.

Este tipo de resolución, desde este punto de vista, más potente para ambos ámbitos parece no lograr consolidarse como una alternativa cierta y creemos que ello está vinculado con el tipo de implicancias sociales más amplias que tiene su abordaje.

La Administración Pública refiere, más allá de los énfasis y perspectivas diversas, al trata- 
miento de lo estatal, de sus objetivaciones institucionales y de la orientación de sus políticas y de su gestión más cotidiana, por lo menos en tanto una de sus improntas constitutivas. En este sentido, ella alude a «lo establecido» y al «establecer» socialmente, desde un imperativo (propio del Estado) que debe presentarse absoluto, inmune a potenciales cuestionamientos y por sobre lo arbitrario o lo particular, para usufructuar de su legitimidad.

Para ponerlo en otros términos, cualquier tipo de producción estatal (v.g. el sentido de sus instituciones, sus normas escritas, los objetivos y el funcionamiento de sus agencias, la actuación de sus responsables, etc.) debe resultar para la sociedad en un sentido general positivo (directo o instrumental), en relación con el bienestar del conjunto de la población y más allá de las múltiples modalidades en que se concrete, porque en ese potencial bienestar se legitima y sostiene, como campo de prácticas, actores y agencias específicas.

Por lo tanto, el poner en cuestión la cosa pública (aún en un sentido intelectual, vinculado a lo reflexivo) resulta ser un asunto socialmente controvertido, cuyas implicancias exceden el alcance formativo de un programa universitario.

La resolución más habitual a esta controversia, paradójicamente, parece consistir en su desconocimiento, el que se concreta en el abordaje de sólo uno de sus polos posibles, en forma bastante excluyente:
- Ya sea, a través de una producción académica crítica pero excesivamente alejada de la perspectiva o posición propia de los niveles político o administrativos.; o

- Anulando cualquier problematización crítica.

La primera, en general, se trata de una reflexión valiosa, pero constitutivamente imposibilitada de aportar a la intervención, porque las exigencias de ésta aparecen descalificadas en tanto tales, dadas determinadas restricciones de orden político-estructural, sobre las que se supone que no es posible operar directamente en términos de gestión.

La segunda, en cambio, se trata de una producción de carácter marcadamente prescriptivo que ofrece respuestas seguras en relación con la adecuación de desempeños específicos, pero que vela el conjunto particular de preguntas o cuestiones sobre las que se inscriben esas estipulaciones.

En este último sentido, sitúa artificiosamente la condición compleja y contradictoria de la posición de políticos y funcionarios, de sus ámbitos institucionales de actuación, de las relaciones sociales involucradas en éstos, o de las condiciones más globales sobre las que se prefigura su desempeño.

Ambos tipos de resoluciones no son caprichosas. Frente a la disyuntiva que involucra tratar la administración pública, se abren primariamente estas dos opciones. Una alternativa transitada, consiste el develar el carácter estructuralmente contradictorio y conflic- 
tivo de lo estatal, de sus instituciones y por lo tanto de las orientaciones de gestión o las prácticas de los funcionarios. En este caso, se trata de denunciar, desde cierto exterior (que puede ser el tradicional académico), esta característica de orden vasto y que remite a una concepción crítica de la sociedad, caracterizada por la asimetría o la desigualdad.

La segunda opción, a manera de negativo, reproduce la concepción predominantemente instituida respecto del Estado en su condición de garante del bienestar común, en un sentido absoluto, donde cualquier indicador de una actuación arbitraria (con implicancias en términos de desigualdades) se interpreta como desvío de una normalidad supuesta, naturalizada, sobre la cual sólo sería necesario intervenir adecuadamente, a través de indicaciones técnicas correctas, para encausarla.

Cabría aclarar, que esta caracterización en tanto opciones extremas, no es reflejo directo de la producción en Administración Pública como especialidad; en realidad, sólo puede sostenerse válidamente en términos de coordenadas que atraviesan el conjunto; es decir, una producción que puede interpretarse y clasificarse fácilmente más próxima a uno u otro extremo. Sin embargo constituye una cuestión que, recreada desde diferentes perspectivas, tiene ya presencia cierta. (v.g. Aguilar Villanueva, 1998: 42 y ss)

Desde nuestra perspectiva es posible construir un curriculum (un texto privilegiante) que sin eludir la problematización de lo estatal y de su administración (o, precisamen- te por eso), sea un aporte significativo para orientar el tipo de intervención posible o deseable en la gestión pública, sobre criterios ético-políticos y no exclusivamente técnicos.

Un camino de producción intelectual capaz de contener ambos polos, en tensión, sin renunciar a las contribuciones propias de uno y otro. Es decir, un «texto» que se intersecta con el contexto primario, eludiendo tanto la asimilación plena a él como su escisión más profunda: una intersección entre la lógica académica y la del campo de la gestión pública; a partir de lo cual es posible generar una nueva.

Creemos que esta última opción, el desarrollo de una perspectiva crítica orientada a la intervención, es posible. Y, esto porque el secreto de este carácter contradictorio de lo estatal es, justamente, contener en simultáneo ambas dinámicas: responder a intereses específicos, particulares, en una sociedad sesgada políticamente pero, simultáneamente, atender el imperativo de ser agente de «bienestar general»o «igualdad». Una lógica paradójica, aún bastante explicativa de sus instituciones y de las prácticas de los diversos actores involucrados en (y con) ellas.

Dar cuenta de esta tensión o paradoja conlleva considerar que necesariamente nos acompaña, ya sea nos encontremos inmersos en el campo de la gestión, donde no existen certezas dadas, absolutas, ni acciones incondicionadas socialmente o políticamente inocuas; o en el campo académico: donde cualquier reflexión abstracta o crítica sobre lo público 
involucra concebirlo de determinada forma y, por lo tanto, esbozar determinadas tendencias y formas adecuadas hacia el «hacer» en él.

Pensar críticamente y hacer sobre lo públicoestatal son opciones diferentes que habitualmente se presentan, por los motivos de peso expuestos, como mutuamente excluyentes. Sin embargo, en alguna medida y con distintos sesgos, la «reflexión» y la «prescripción» siempre son susceptibles de remitirse.

Pensamos que esta zona compartida, pero de límites móviles, frágiles y ciertamente poco apacible, esta aún disponible y guarda una especial fecundidad para la definición y el desarrollo de las propuestas de formación en Administración Pública.

Finalmente, una última cuestión que se señala respecto de la construcción del curriculum en Administración Pública, refiere al análisis de su condición disciplinar.

Como se menciona, se trata de una cuestión abierta, poco consolidada y, en tanto tal, significativa respecto de la construcción de un programa de formación (el texto o el contexto secundario) y de su fundamento más amplio (el contexto primario).

La propia definición de esta cuestión- ¿cuál es el carácter disciplinar de la Administración Pública?- supone ya que esta especialidad, en tanto tal, conlleva cierta base disciplinaracadémica. Aunque avanzar sobre este tema resulta incierto y, probablemente, lo más consensuado al respecto sea: i) que esta especia- lidad no involucra el desarrollo de una única disciplina y; ii) que tampoco se conforma como un área específica subsumida.

Tomemos por caso, la siguiente definición de la especialidad:

Considerar a la administración y las políticas públicas como un objeto de estudio, más que como una disciplina. En tal sentido, su estudio estaría abierto a todo tipo de profesionales que compartan el objetivo básico de considerar que su enseñanza, investigación y transformación constituyen actividades valiosas (INPAE, Lineamientos Estratégicos).

Desde esta perspectiva, la especialidad se define a partir de su objeto de estudio, el cual convoca diferentes «profesionales». O sea, es el propio objeto, el centro unificador, constitutivo e integrador del conjunto. A su vez, esta definición en alguna medida expresa esta condición compartida que tiene entre el campo académico (que hace de la administración pública un objeto de estudio) pero que para tal fin involucra a «todo tipo» de profesionales (su adscripción al campo profesional).

Sin embargo, el problema de su condición disciplinaria no queda totalmente resuelto, (más allá de suponer lógicamente que debe contar con alguna base de este tipo y que no es única). Se podría inferir entonces que una acepción bastante generalizada es su condición multidisciplinar. Pero la cuestión realmente abierta es: qué disciplinas diversas la constituyen, en qué tipo de relaciones, con 
qué criterios de convergencia.

Como se ha desarrollado, la definición del texto, conlleva el establecimiento de una serie de regulaciones y estructuras que, de por sí, también configuran el campo de la especialidad. A su vez, siempre implica una selección, una operación de inclusión/exclusión, privilegiar determinados contenidos sobre otros, con implicancias de carácter político e institucional. (De Alba, 1994; Beillerot, 1998; Cols, 2001).

Es decir, estos distintos componentes de bases disciplinarias diversas, ya sea considerando la delimitación de la especialidad o del curriculum de formación, no ocupan una posición simétrica o equivalente, simplemente agregada horizontalmente; se estructuran a partir de determinados criterios, determinadas preocupaciones, más propias de una impronta disciplinar que de otra. Es un campo en tensión y movimiento, con consecuencias también epistemológicas.

Así, por caso, si es el Derecho y no las Ciencias Políticas, el que guarda un lugar privilegiado en este conjunto multidisciplinar, los sentidos respecto del estudio de lo legal -por ejemplo- como sesgo general de la carrera o de la perspectiva de estudio serán cualitativamente diferentes. Es muy probable que estén orientadas hacia lo prescriptivo-valorativo (según las reglas del «Derecho», como disciplina $y$ práctica). Mientras que, si la situación fuese inversa, es probable que esta preocupación por lo legal tuviese un sesgo más analítico y quedara redefinida en nuevos términos tales como una preocupación por la regulación de sistemas sociales, por la institucionalidad, etc.

En definitiva, parece importante en el trabajo de producción o análisis curricular identificar o explicitar cuál es esa configuración multi o inter-disciplinar específica, cuáles esos criterios de jerarquización y de yuxtaposición, cómo son los diversos predominios relativos posibles de disciplinas o sub-disciplinas y las elecciones (expresas o tácitas) producidas.

\section{Conclusión}

En este trabajo se abordó el problema de la formación superior en Administración Pública, desde una preocupación especial por lo curricular, considerado en tanto estructuración institucional, de múltiples consecuencias, no sólo respecto de la enseñanza de la especialidad sino de su configuración misma, en tanto tal.

Así, pensar en la creación o en el desarrollo de Programas formativos involucra tratar un conjunto de aspectos significativos que si bien no se presentan directamente en un plano técnico u operativo, respecto de la definición, revisión o evaluación de estos Programas; están también allí presentes, en términos de implicancias, condiciones y posibilidades de carácter «menos práctico» pero de vastos efectos.

Las transformaciones en la historia reciente de la educación superior, generalizables a la Región, se yuxtaponen a una tradición previa, universitaria o disciplinar-académica que no desaparece, sino que permanece aún vigente, 
en tanto universo autocontenido, con vitalidad propia que se articula, de forma variable (confrontado, agregado o en complementariedad) a nuevas tendencias que aparecen en forma progresiva, aunque poco ordenada $y$ desigual, según los diferentes escenarios institucionales o nacionales que se consideren.

Estos cambios, que hemos caracterizado en un sentido general como el arribo de una matriz "particularista», se expresan en la diversificación de las conformaciones y naturalezas de las instituciones del sector; en la construcción de relaciones competitivas entre ellas, sobre las que se trazan distintos tipos de asociaciones; en una creciente diferenciación interna respecto de las características y coordinación de las unidades que las componen; en la presencia creciente de la política gubernamental o de organizaciones internacionales que traccionan estas tendencias, y que simultáneamente asumen como propia la función de regulación de un conjunto diversificado, variado y segmentado.

Ellas pueden apreciarse como condiciones de producción relevantes respecto de la construcción de propuestas formativas. Lo que hemos denominado campo re-contextualizador del curriculum. Más aún, en este campo es posible identificar una presencia creciente de inquietudes de orden educativo, que traen al centro de la escena la actividad de enseñanza y el interés por lo curricular.

Por su parte, la consideración de los desarrollos curriculares, en términos de análisis teórico: su consideración en términos de una relación, en tensión, entre un texto y un contexto, permite poner en nuevos términos la comprensión y el sentido de definiciones que pueden presentarse en la realidad institucional más cotidiana como coyunturales, soluciones de necesidad, invenciones totalmente independientes o exclusivamente sujetas a la política institucional o una lógica racional. Cualquiera de estas respuestas puede ser legítimamente cierta, pero a ellas habría que agregarle este otro plano que redefine en profundidad esa experiencia y puede adelantar ciertas limitaciones ( $\mathrm{y}$ también ciertas posibilidades) de orden más estructural, dadas por ese tipo de relación compleja entre contextos de producción y reproducción, del que participa cualquier iniciativa de esta naturaleza.

Por último, el abordaje respecto de la definición de la orientación formativa predominante, en relación con los sesgos profesionales y académicos propios de la Administración Pública como especialidad, constituye un material que habilita pensar en nuevas posibilidades de orden ético y político sobre las cuales desplegar la reconfiguración de las carreras del área, pero también el área misma. A su vez, la consideración del carácter multidisciplinar de la especialidad, intenta dar visibilidad a las numerosas implicancias, especialmente de orden epistemológico, respecto de cualquier tipo de ordenación que pueda adquirir esa multiplicidad y variedad potencial. 


\section{Referencias}

Agoff, S. y otros (2004). Construcción del saber administrativo en la Argentina: una aproximación operacional. Buenos Aires. Instituto de Investigaciones Administrativas. FCE.UBA.

Aguilar. (1998). Administración y Estado moderno. Notas para una discusión en VVAA. La política como respuesta al desencatamiento del mundo, Eudeba. Buenos Aires.

Alonso, M. (2008, julio/septiembre). El sentido de la universidad a través de sus transformaciones en la historia reciente. Revista de Educación Superior, Vol. 37 (147). México.

Beillerot, J. y otros (1998). Saber y relación con el saber. Paidos. Buenos Aires.

Berstein, B. (1993). La estructura del discurso pedagógico. Morata. Madrid.

Cols, E. (2001). El curriculum como institución y la dimensión institucional de los procesos curriculares. Revista Espacios. Año VIII (21). UNPA. Río Gallegos.

De Alba, A. (1994). Curriculum: crisis, mito y perspectivas. México. UNAM- CESU.

Díaz, J. y otros (2002). Univeridades e Avalidacao: entre a ética e o mercado. Editora Insular. Florianópolis.

Fernández, N. (2003). La educación superior argentina en debate. Situación, problemas y perspectivas. Buenos Aires. EUDEBA.

Fernández, N.; Vales, A. y Alonso, M. (2004). Saber administrativo y Educación. Buenos Aires. Instituto de Investigaciones Administrativas. FCE.UBA.

De Fanelli, A. (1996). La reforma de la educación superior en la Argentina. Entre el mercado, la regulación estatal y la lógica de las instituciones. Revista Paraguaya de Sociología, No 97, Asunción, Paraguay.

De Fanelli, A. (1998). La universidad pública frente a la nueva lógica de las políticas públicas y del mercado. Buenos Aires. (Mimeo). CONICET-CEDES.

Gibbons, M. (1999). La nueva producción de conocimiento. Pomare- Corredor. Madrid.

INPAE- Lineamientos estratégicos. (2004). Recuperado de: http://redinpae.org/

Kent, R.; (comp.) (1996). Los temas críticos de la educación superior en América Latina. Estudios comparados, vol. I y II. FCE. México.

Klein L. y Sampaio, H. (1996). Actores, arenas y temas básicos. Kent, R. (comp.) Los temas crí- 
ticos de la educación superior en América Latina. Estudios Comparados. FCE. México

Krotsch, P. (2000a). La universidad frente a los desafíos de la imprevisibilidad y la integración social. Revista Avalidacao. San Pablo. Vol. 5 (1).

Krosch, P. (2000b). Sistemas educativos y Reformas Comparadas. Universidad Nacional de Quilmes. Buenos Aires.

Lundgren, U. (1992). Teoría del curriculum y escolarización. Morata. Madrid.

Reid, W. (1999). Curriculum as institution and practice. Erlbaum Associates. New Jersey.

Suárez, F. (2001). Marco conceptual. Documento de trabajo del Proyecto «Saber Administrativo». SECyT. FCE. UBA. Buenos Aires.

Suárez, F.; Agoff, S.; Contreras, M. y Padlog, M. (2000). El saber administrativo y la profesión del administrador. Encuentro del Consejo Profesional de Ciencias Económicas. Mimeo. Buenos Aires.

Suárez F y Felcman I (1975). Tecnología y organización: un aporte para el análisis de las tecnologías administrativas. El Coloquio. Buenos Aires

Suárez, Felcman, Losano y Zonis (2004). El saber administrativo como tecnología. Instituto de Investigaciones Administrativas. FCE.UBA. Buenos Aires

Thompson, J. (1967). Organization in action: Social sciencie bases of administrative theory. Mc. Graw Hill Book. USA. 